\title{
Focal solitary necrotic nodules in fatty liver: characteristics on conventional and contrast-enhanced ultrasonography
}

\author{
Yue-ling Peng ${ }^{1} \cdot$ Li-ping Liu $^{2} \cdot$ Yan-jing Zhang ${ }^{2} \cdot$ Jing-jing Liu ${ }^{2} \cdot$ Xiao-Ling Yu ${ }^{3}$
}

Received: 30 July 2021 / Accepted: 19 October 2021 / Published online: 29 January 2022

(C) The Author(s) 2022

\begin{abstract}
Purpose Focal lesions in fatty liver are difficult to diagnose using conventional ultrasonography (CVUS). The aim of this study was to investigate the characteristics of solitary necrotic nodules (SNNs) in fatty liver using CVUS and contrastenhanced ultrasonography (CEUS) and to evaluate the diagnostic value of CEUS for SNNs in fatty liver.

Methods Fifteen SNNs in the fatty liver of fifteen patients were examined by both CVUS and CEUS. The contrast agent SonoVue was used for CEUS. The characterization and shape of these SNNs in the fatty liver were analyzed using CEUS.

Results CVUS revealed eight oval-shaped, six irregularly shaped, and one wedge-shaped SNN in the fatty liver. The six irregularly shaped lesions on CVUS were revealed to comprise four gourd-shaped, one serpiginous, and one 3-pin socketshaped nodule on CEUS. One of these SNNs showed a subcapsular wedge shape, with peripheral and distinct internal septal hyperenhancement in the arterial phases that washed out in the portal phase; moreover, most areas of th lesion showed no internal enhancement in any of the three phases. Fourteen of the lesions were characterized as lacking internal enhancement, and 12 of them had mild-moderate peripheral thin enhancement in the arterial phases. Twelve of the 15 nodules could be considered for diagnosis as SNNs by CEUS, which was further proved by US-guided biopsy and histopathology. However none of them could be considered for diagnosis as SNNs by CVUS.

Conclusions CEUS is a valuable tool for visualizing the characteristics of SNNs in fatty liver to improve the differential diagnosis.
\end{abstract}

Keywords Solitary necrotic nodule $\cdot$ Fatty liver $\cdot$ Contrast agent $\cdot$ Ultrasonography

\section{Introduction}

The incidence of fatty liver has increased over the past 2 decades; in fact, $25 \%$ of the world's population is currently thought to have nonalcoholic fatty liver disease (NAFLD) [1], and there is even a high prevalence in children $[2,3]$. Ultrasonography is the first-line imaging modality for fatty liver and focal liver lesions. However, focal lesions in the fatty liver are difficult to diagnose using conventional

Xiao-Ling Yu

yux10182@outlook.com

1 Department of Nephrology, Shanxi People's Hospital, Taiyuan 030001, China

2 Department of Ultrasound, First Hospital of Shanxi Medical University, Taiyuan 030001, China

3 Department of Interventional Ultrasound, Chinese People's Liberation Army General Hospital, 28 Fuxing Rd, Beijing 100853, China ultrasonography (CVUS) [4, 5] and usually do not show the typical characteristics of malignant and benign lesions; for example, there may be no hypoechoic halo sign typical of malignant tumors and nonhyperechoic lesions of hemangiomas.

Solitary necrotic nodules (SNNs) of the liver was first described by Shepherd and Lee [6]. The etiology of SNNs of the liver is still unknown despite multiple hypotheses, including trauma [6], parasitic infestation [6,7] and sclerotic hemangioma [6, 8], and allergic and defense reactions for a history of gastrointestinal cancer [9]. SNN of the liver is a rare and non-neoplastic lesion with a very challenging diagnosis $[10,11]$. Studies on features on MRI, CT and contrast-enhanced ultrasonography (CEUS) of SNNs have been reported, but they are still frequently misinterpreted as malignant to surgical resection. Recently, it was reported that an SNN was misdiagnosed as metastasis by CT MRI and PET-CT and removed surgically. Finally, the SNN was proved by pathology to have a completely necrotic core and 
a fibrotic capsule containing infiltrating inflammatory cells [12]. Therefore, there is a need to continue further research on SNNs to improve the diagnostic awareness.

Low mechanical index (MI) real-time CEUS has greatly improved the differential diagnosis of focal liver lesions $[5,13-15]$. Because of the increasing incidence of focal lesions in patients with fatty liver in recent years, some cases of SNNs in the liver have been collected since we have researched focal lesions in fatty liver for many years. We describe and analyze the characteristics of SNNs in fatty liver using CVUS and CEUS in this study to improve their diagnosis.

\section{Materials and methods}

\section{Patients}

During a 7-year period, a total of 15 patients with SNN lesions in the fatty liver were investigated using CEUS. There were 11 men and 4 women with a mean $( \pm$ SD) age of $46.73 \pm 7.32$ years (34-58 years). There was no history of hepatitis B or HCV infection in any of these patients. In these patients, ultrasound examination found lesions, such as ten patients for health checkups; four patients for upper abdominal discomfort, including one with cholecystitis and gallbladder polyps; and one patient for postoperative followup of gastric cancer. One patient had alcoholism, 4 of whom had elevated aminotransferase.

The mean diameter of the lesions was $2.06 \pm 0.89 \mathrm{~cm}$ (from 0.85 to $4.60 \mathrm{~cm}$ ). The ultrasonographic diagnosis of fatty liver was established based on typical ultrasonographic findings: a markedly echogenic liver compared with the right kidney. In our study, SNNs and fatty livers were confirmed by ultrasound-guided biopsy. Written informed consent was provided by each patient to participate in research and to publish, and the study was approved by the ethics committee of Chinese People's Liberation Army General Hospital and the first Hospital of Shanxi Medical University,

\section{CVUS}

Ten of the patients were imaged using a Sequoia 512 scanner (Siemens Medical Ultrasound, Mountain View, CA) equipped with contrast pulse sequencing (CPS) software, allowing for a real-time depiction of blood perfusion in the lesion at a low MI. A 4V1 vector transducer with a frequency range of 1-4 $\mathrm{MHz}$ was used. The remaining five patients were assessed using a Mylab_90 scanner (Esaote, SpA. Genoa, Italy), which has a CA431 vector transducer with a frequency range of $1-8 \mathrm{MHz}$ equipped with contrasttuned imaging (CnTI) software.
Baseline ultrasonography of the liver was performed to identify focal lesions, and the location, size, shape, margin, and posterior echo enhancement of the lesion were recorded. The pulse repetition frequency was set to the lowest value that was free of motion artifacts to maximize the color signal of slow-velocity blood flow.

\section{CEUS}

The contrast agent used in this study was SonoVue (Bracco, Milan, Italy), which was supplied as a lyophilized powder and reconstituted with $5 \mathrm{~mL}$ of saline to form a homogeneous microbubble suspension. All patients provided full informed consent prior to receiving the SonoVue injection. CEUS studies were performed after the administration of $2.4 \mathrm{~mL}$ of SonoVue into an antecubital vein in a bolus via a 20-gauge needle followed by a flush with $5 \mathrm{~mL}$ of normal saline.

CPS or CnTI was activated following the injection of the contrast agent. The MI value displayed on screen ranged from 0.13 to 0.17 for CPS and was 0.7 for CnTI. A dualimage real-time display was used to help locate the target lesion during the examination. Digital cine clips of typical CVUS images and all CEUS images were stored on the hard disks of the imaging system and transferred to personal computers for subsequent analysis.

\section{Imaging analysis}

CVUS images and dynamic CEUS images were analyzed by two experienced radiologists (L.P.L. and X.L.Y.) in consensus. The CVUS and CEUS characteristics were recorded.

\section{Results}

\section{Characteristics of the 15 SNNs in the fatty liver on CVUS}

Twelve lesions were found in the right liver lobe, and three lesions were found in the left lobe. All SNNs in the fatty liver were hypoechoic lesions. Nine lesions had homogeneous internal echoes; All others had heterogeneous patterns. CVUS revealed eight oval-shaped, six irregularly shaped, and one wedge-shaped SNN. Eleven nodules had distinct margins, and the other four had indistinct margins. Four nodules had posterior echo enhancement. (Table 1).

Only one wedge-shaped lesion located subcapsularly exhibited peripheral blood vessels on color Doppler flow imaging (CDFI), and the remaining fourteen nodules showed no color signal.

Using CVUS, only four lesions were determined to be benign; however, none of them could be diagnosed as SNNs. 
Table 1 Conventional and Contrast-enhanced ultrasonography Characteristics of 15 SNNs

\begin{tabular}{|c|c|c|c|c|c|c|c|c|c|}
\hline \multirow[t]{2}{*}{ No. } & \multirow[t]{2}{*}{ Sex/age } & \multirow[t]{2}{*}{ Location } & \multirow[t]{2}{*}{ Size $(\mathrm{cm})$} & \multicolumn{4}{|c|}{ 6794525082500CVUS } & \multicolumn{2}{|c|}{ 10223525781000CEUS } \\
\hline & & & & Lesion margin & Internal echo & $\begin{array}{l}\text { Posterior echo } \\
\text { enhancement }\end{array}$ & Shape & Lesion margin & Shape \\
\hline 1 & $\mathrm{~F} / 58$ & $\begin{array}{l}\text { Right anterior } \\
\text { superior seg- } \\
\text { ment }\end{array}$ & 2.0 & Indistinct & Homo & Absent & Irregular & Distinct & serpiginous \\
\hline 2 & $\mathrm{M} / 45$ & $\begin{array}{l}\text { Right posterior } \\
\text { segment }\end{array}$ & 2.5 & Distinct & Homo & Absent & Irregular & Distinct & $\begin{array}{l}\text { 3-pin socket- } \\
\text { shaped }\end{array}$ \\
\hline 3 & $\mathrm{~F} / 49$ & $\begin{array}{l}\text { Right posterior } \\
\text { segment }\end{array}$ & 1.3 & Distinct & Homo & Absent & Oval & Distinct & Oval \\
\hline 4 & $\mathrm{~F} / 50$ & $\begin{array}{l}\text { Right posterior } \\
\text { segment }\end{array}$ & 2.3 & Distinct & Homo & Absent & Irregular & Distinct & Gourd-shaped \\
\hline 5 & $\mathrm{~F} / 55$ & $\begin{array}{l}\text { Right anterior } \\
\text { inferior segment }\end{array}$ & 1.3 & Distinct & Homo & Absent & Oval & Distinct & Oval \\
\hline 6 & $\mathrm{M} / 38$ & $\begin{array}{l}\text { Left lateral seg- } \\
\text { ment }\end{array}$ & 0.85 & Distinct & Homo & Absent & Oval & Distinct & Oval \\
\hline 7 & $\mathrm{M} / 43$ & $\begin{array}{l}\text { Right anterior } \\
\text { superior seg- } \\
\text { ment }\end{array}$ & 1.8 & Distinct & Heter & Absent & Oval & Distinct & Oval \\
\hline 8 & M/44 & $\begin{array}{l}\text { Right anterior } \\
\text { segment }\end{array}$ & 1.2 & Distinct & Homo & Absent & Oval & Distinct & Oval \\
\hline 9 & $\mathrm{M} / 55$ & $\begin{array}{l}\text { Right posterior } \\
\text { segment }\end{array}$ & 2.45 & Distinct & Homo & Absent & Irregular & Distinct & Gourd-shaped \\
\hline 10 & $\mathrm{M} / 34$ & $\begin{array}{l}\text { Right anterior } \\
\text { inferior segment }\end{array}$ & 4.6 & Distinct & Heter & Slight enhancing & Wedge & Distinct & Wedge \\
\hline 11 & $\mathrm{M} / 45$ & $\begin{array}{l}\text { Right anterior } \\
\text { inferior segment }\end{array}$ & 2 & Distinct & Heter & Absent & Oval & Distinct & Oval \\
\hline 12 & M/56 & $\begin{array}{l}\text { Right posterior } \\
\text { segment }\end{array}$ & 1.1 & Indistinct & Heter & Absent & Irregular & Distinct & Gourd-shaped \\
\hline 13 & $\mathrm{M} / 45$ & $\begin{array}{l}\text { Left lateral seg- } \\
\text { ment }\end{array}$ & 1.8 & Distinct & Heter & Slight enhancing & Oval & Distinct & Oval \\
\hline 14 & $\mathrm{M} / 48$ & $\begin{array}{l}\text { Right anterior } \\
\text { inferior segment }\end{array}$ & 2.6 & Indistinct & Heter & Slight enhancing & Irregular & Distinct & Gourd-shaped \\
\hline 15 & $\mathrm{M} / 36$ & $\begin{array}{l}\text { Left lateral seg- } \\
\text { ment }\end{array}$ & 1.76 & Indistinct & Homo & Slight enhancing & Oval & Distinct & Oval \\
\hline
\end{tabular}

\section{Characteristics of SNNs in fatty liver on CEUS}

On CEUS, eight of the SNNs were oval shaped, and one was wedge shaped. The six irregularly shaped lesions on CEUS were revealed to comprise four gourd-shaped, one serpiginous, and one 3-pin socket-shaped nodule using CEUS. All lesion margins were distinct on CEUS. (Figs. 1, 2, 3, Table 1).

Twelve of the SNNs showed mild-moderate peripheral thin enhancement in the arterial phase, which became identical to the signal in the liver parenchyma in the portal and delayed phases with no enhancement in the interior of the lesion (Fig. 1). One wedge-shaped SNN was characterized by peripheral and distinct internal septal hyperenhancement in the arterial phases, and hypoenhancement in the portal and delayed phases (Fig. 2), moreover, most areas of this lesion showed no internal enhancement in any of the three phases; otherwise, the other nodules showed no interior enhancement. The other two lesions had no enhancement in any of the three phases.

Twelve lesions could be considered for the diagnosis of SNNs using CEUS, which was further proven by USguided biopsy and histopathology. The smallest lesion could still not be determined to be a cyst or SNN due to the lack of interior enhancement in all three phases with no thin peripheral enhancement in the arterial phase; subsequently, this lesion was diagnosed as an SNN by USguided biopsy and histopathology (Fig. 3). One wedgeshaped lesion was thought to be an inflamed and possibly infected lesion but was proven by US-guided biopsy and histopathology later to be an SNN (Fig. 2). 
Fig. 1 Focal hypoechoic lesion (SNN) in a 48-year-old man with fatty liver. A Sonogram showing the focal irregular hypoechoic lesion (arrow) in the right lobe of the fatty liver; B Contrast-enhanced image acquired with CPS showing mild-moderate peripheral enhancement in a gourd-shaped lesion in the arterial phase (left arrow), with no enhancement within the lesion, corresponding to the hypoechoic lesion (right arrow) seen in the fatty liver on the baseline sonogram; $\mathbf{C}$ No enhancement was seen within the lesion in the portal phase, corresponding to the hypoechoic lesion (right arrow) seen in the fatty liver on the baseline sonogram; D Histology of the liver biopsy specimen showing central coagulation necrosis surrounded by fibrous tissue with infiltrating inflammatory cells (hematoxylin-eosin, original magnification $\times 200$ ); $\mathbf{E}$ Histology of biopsy specimen of the surrounding hepatic parenchyma showing steatosis and balloon cells (hematoxylin-eosin, original magnification $\times 200$ )
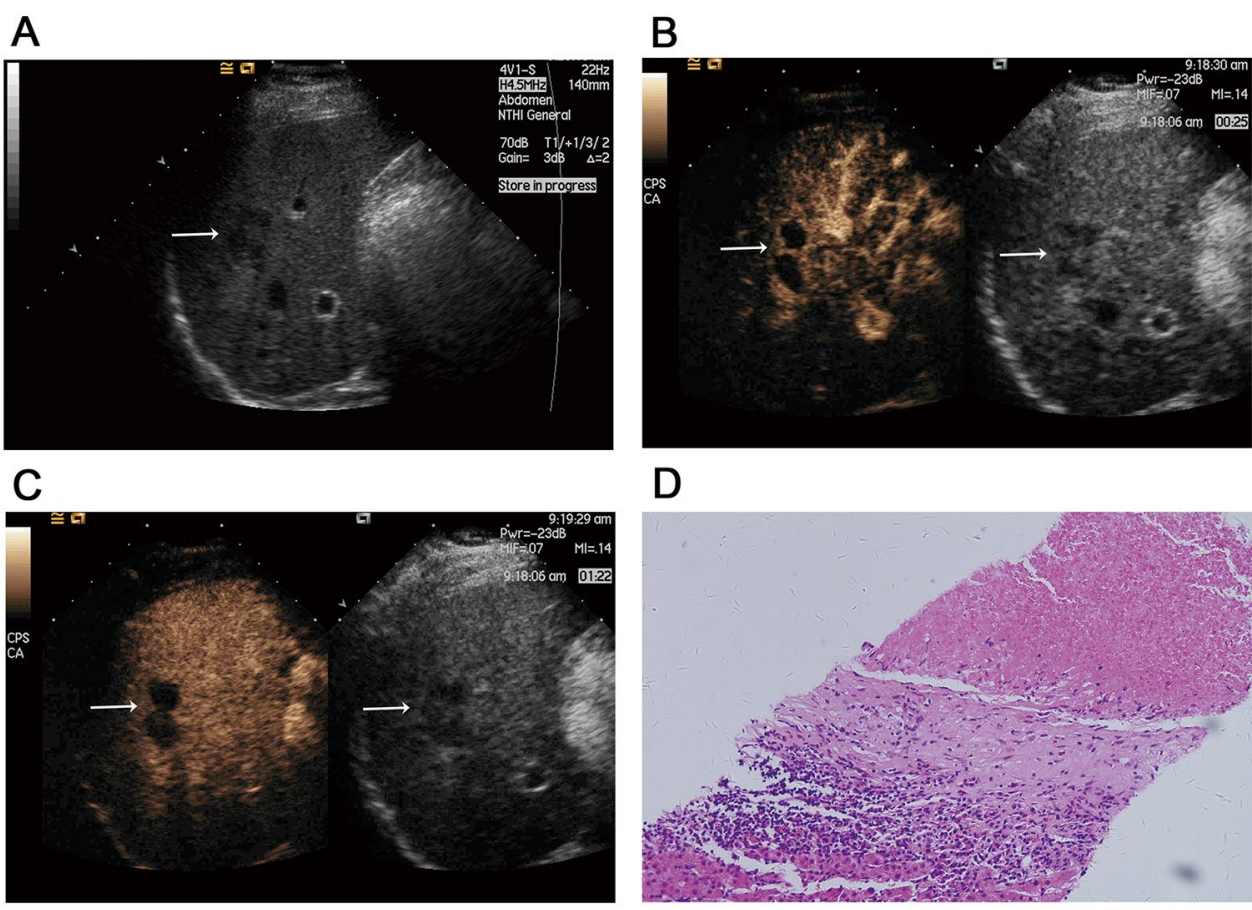

D

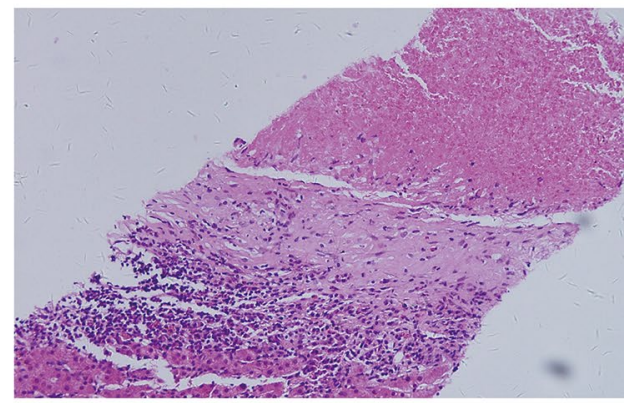

E

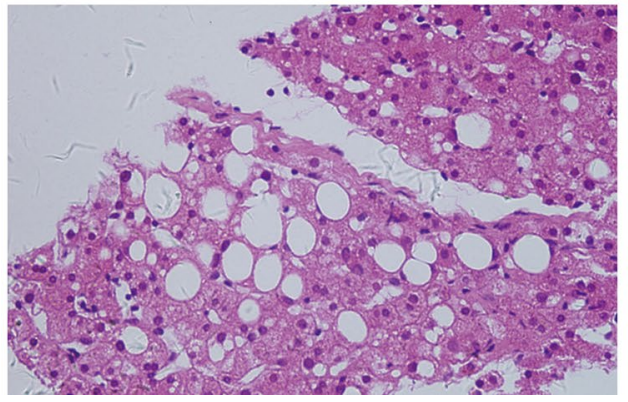

\section{Discussion}

SNN of the liver is usually benign. The histopathology of SNN demonstrates central coagulation necrosis with a peripheral fibrous capsule and infiltration of inflammatory cells. Most patients generally had no clinical symptoms, which were accidentally detected during ultrasound examination. Because of inadequate knowledge of SNN of the liver, it is often interpreted as malignant, leading to surgery. Chen et al. [16] reported one SNN of the liver that was indeterminate on both abdominal CVUS and MRI, while F-18 fluorodeoxyglucose positron emission tomography/computed tomography (F-18 FDG PET/ CT) showed a solitary hypermetabolic mass in the liver; therefore, malignancy was suspected, and postoperative pathology showed an SNN with larval infestation. De Luca et al. [17] reported one SNN of the liver with compression of the celiac axis (Dunbar syndrome); after surgery, the pathological diagnosis of this focal lesion was SNN due to ischemic injury.
In our study, two SNNs with likely hypoechoic halos were difficult to distinguish from hepatic metastasis using CVUS; however, they were diagnosed using CEUS. In our study, all SNNs were hypoechoic against a fatty liver background on CVUS, four lesions had posterior echo enhancement, and four were characterized by indistinct margins. Using CVUS, only four lesions were determined to be benign, but none of them could be diagnosed as SNNs. Twelve of fifteen lesions were correctly considered for the diagnosis of SNNs using CEUS, which was further proven by US-guided biopsy and histopathology.

SNNs can often be easily misdiagnosed as malignant lesions of the liver. Yoon et al. [18] reported that two SNNs of the liver mimicked metastasis on radiologic images. One of the lesions in arterial- and portal-phase spiral CT showed focal wall thickening with enhancement in the fundus of the gallbladder and a $1.0 \mathrm{~cm}$ ovoid hypoattenuating nodule with peripheral rim enhancement in the right lobe of the liver, which suggested gallbladder cancer with hepatic metastasis. After surgery, histopathology revealed that the gallbladder 
Fig. 2 Focal hypoechoic lesion (SNN) in a 34-year-old man with fatty liver. A Sonogram showing the focal wedge-shaped hypoechoic lesion (arrow) in the subcapsular region of the fatty liver; B contrast-enhanced image acquired with CPS showing peripheral and septal enhancement, with no internal enhancement during the arterial phase (left arrow), corresponding to the hypoechoic lesion (right arrow) seen in the fatty liver on the baseline sonogram; C contrast-enhanced image acquired with CPS showing a likely SNN with wash out in the portal phase, corresponding to the hypoechoic lesion (right arrows) seen in the fatty liver on the baseline sonogram; D histology of biopsy specimen showing coagulation necrosis (hematoxylin-eosin, original magnification $\times 200$ ); $\mathbf{E}$ histology of biopsy specimens showing fibrous tissue with infiltrating inflammatory cells (hematoxylin-eosin, original magnification $\times 200$ ); $\mathbf{F}$ biopsy specimen confirming the surrounding hepatic parenchyma as fatty liver with steatosis and balloon cells (hematoxylin-eosin, original magnification $\times 200$ )
A

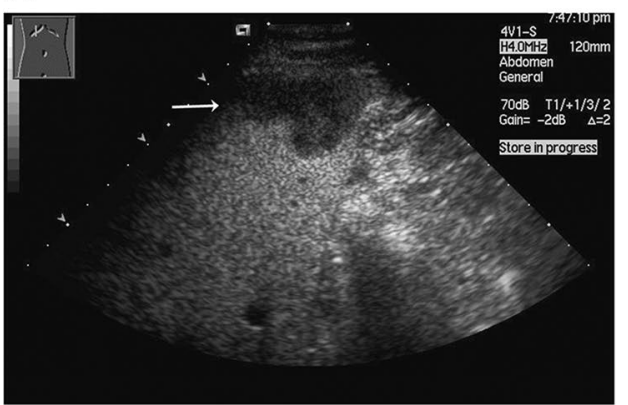

C

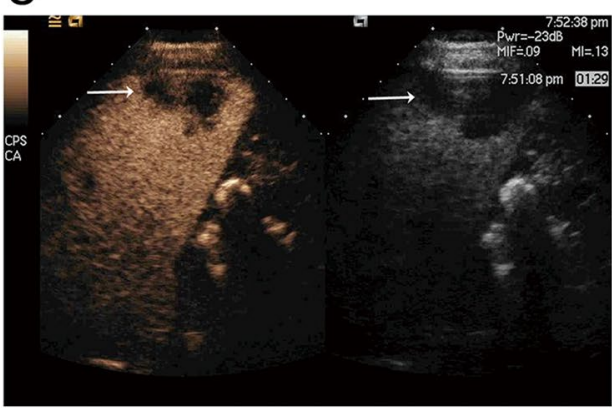

E

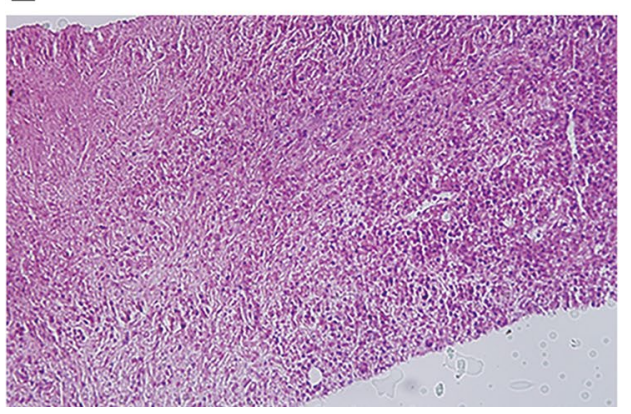

B

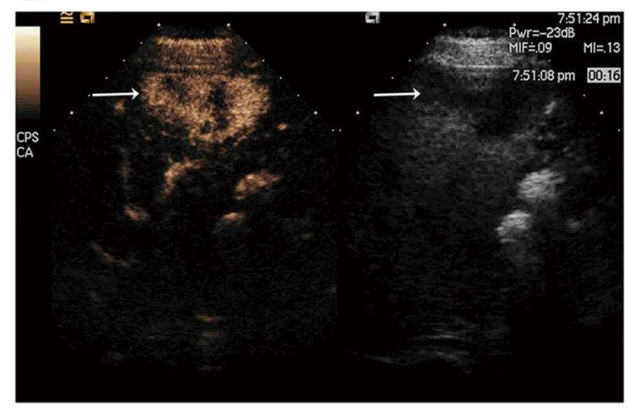

D

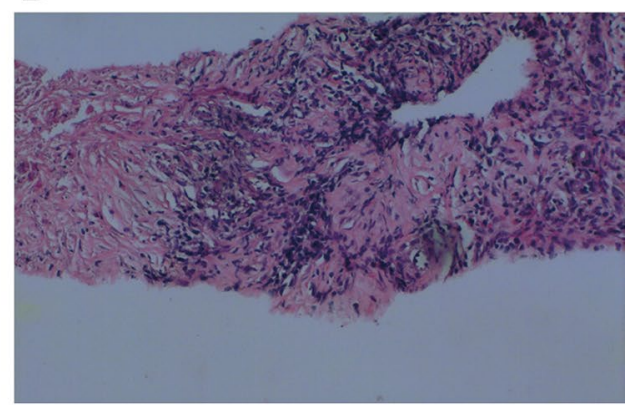

$\mathrm{F}$

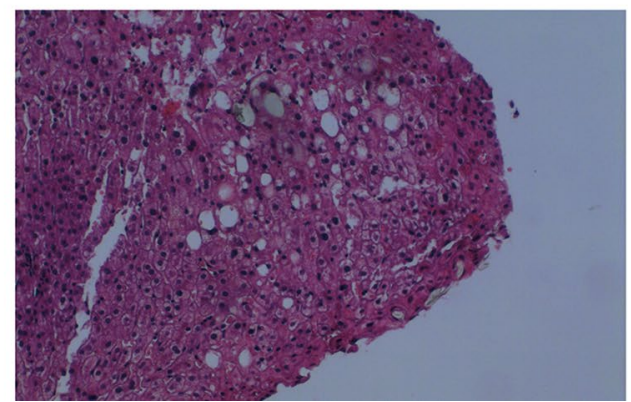

lesion was an adenocarcinoma; however, the hepatic lesions were SNNs composed of a central necrotic core and a peripheral fibrotic capsule with inflammatory cells.

Delis et al. [19] reported a $7 \mathrm{~cm}$ hyperechoic lesion in the right liver lobe using abdominal US. Contrast-enhanced CT demonstrated encapsulation with peripheral enhancement, a necrotic core and contrast washout in the venous phase, which led to a suspicion of adenoma or hepatocellular carcinoma (HCC), although fever and leukocytosis suggested a possible infectious process. The patient was treated with intravenous antibiotics for 7 days until the fever subsided and the complete white cell count normalized. Due to the high suspicion for a malignancy, postoperative pathology was performed and indicated a hemorrhagic infarct and extensive hepatocellular necrosis due to sinusoidal portal vein thrombosis.

Wang et al. [20] reported 17 SNNs in the liver, of which eight were oval, and the others were lobulated. However, the actual shapes of some SNNs in the fatty liver cannot be easily visualized on CVUS. In our study, six lesions were characterized as irregularly shaped on CVUS. Using CEUS, we found that four were gourd shaped (Fig. 1), one was serpiginous shaped, and one was 3-pin socket-shaped. CEUS distinctly revealed the shape and margin of SNNs in the fatty liver, and some of these lesions exhibited typical SNN morphology, such as a gourd shape. Pananwala et al. [7] reported three SNNs of the liver that had a serpiginous shape with areas of necrosis.

Chunyu Lu et al. [21] reported that 13 SNNs (13/24, $54.1 \%$ ) presented with peripheral thin rim-like enhancement in the arterial phase, isoenhancement in the portal phase and delayed phase with no enhancement in the interior of the lesions during three phases. Twelve of the SNNs in our study showed mild-moderate thin peripheral enhancement in the arterial phase, which is similar to the rim enhancement reported by Kim for SNNs of the liver in the arterial phase of MRI [22]. Francica et al. [23] reported that 10 SNNs (10/24, 41.6\%) occurred thin, uniform, hyperenhancing ring in the early arterial on CEUS, but 2 nodules $(2 / 7,28.6 \%)$ showed late rim enhancement in contrasted-enhanced MRI. 
Fig. 3 Focal hypoechoic lesion (SNN) in a 37-yearold man with fatty liver. A Sonogram showing the focal hypoechoic lesion (arrow) in the left lobe of the fatty liver; B contrast-enhanced image acquired with CPS showing no enhancement in the lesion (left arrow) through all three phases, corresponding to the hypoechoic lesion (right arrow) seen in the fatty liver on CVUS. C US-guided biopsy of the lesion; D histology of biopsy specimen showing necrosis surrounded with a fibrotic capsule and infiltration of inflammatory cells (hematoxylin-eosin, original magnification $\times 200$ ); $\mathbf{E}$ histology of biopsy specimen showed the surrounding hepatic parenchyma as fatty liver with steatosis and balloon cells (hematoxylin-eosin, original magnification $\times 200$ )
A

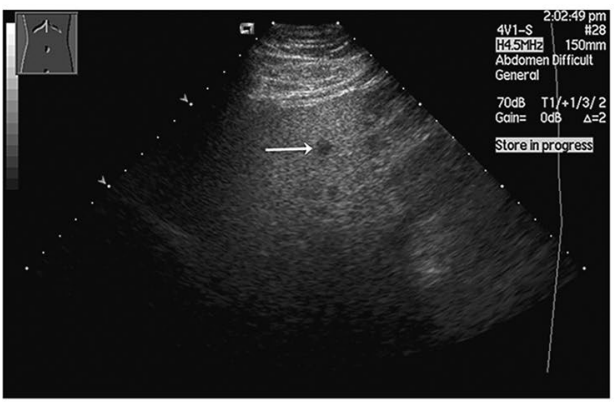

C

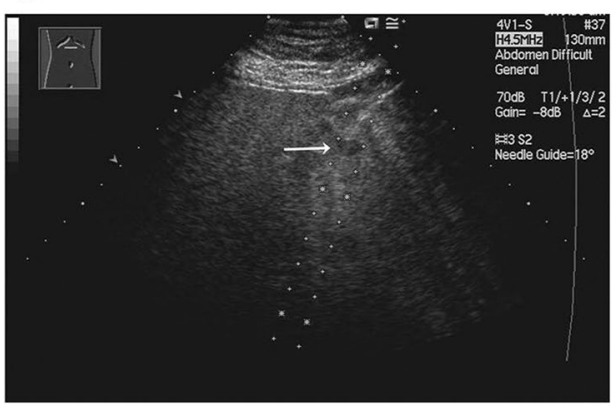

E

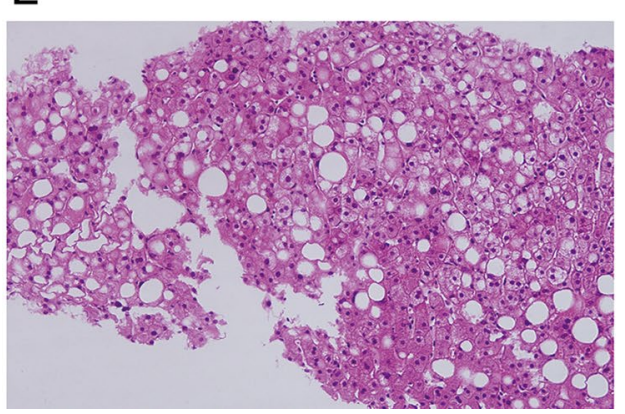

B

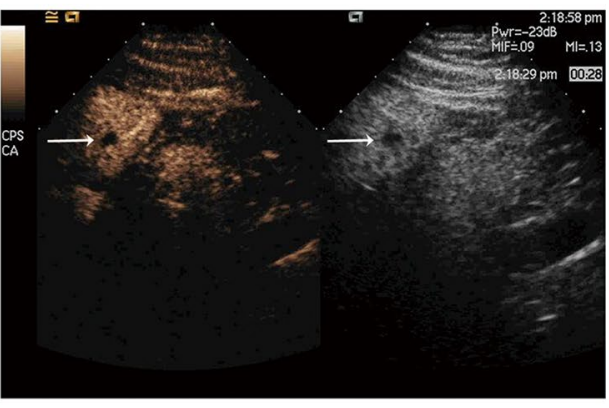

D

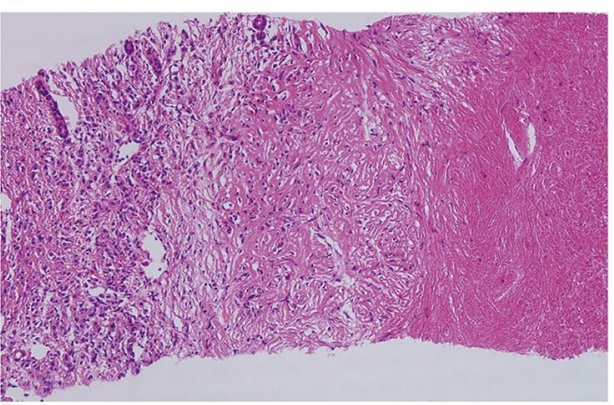

Fang et al. [24] reported SNNs in the liver with a prolonged delayed MRI time; when it was up to $1 \mathrm{~h}$, all lesions represented moderate/marked peripheral enhancement with internal hypointensity. There is a difference between MRI contrast agents and ultrasound contrast agents. The nonspecific contrast agent in the extracellular space used in MRI, such as the delayed enhancement of the thin ring edge, may be the result of the slow diffusion, penetration and clearance of the contrast agent in the wide extracellular space. SonoVue is a blood pool contrast agent, that will not diffuse and penetrate the cell space and is usually excreted with breathing for $6 \mathrm{~min}$. The pathological basis of peripheral thin enhancement in the arterial phase on CEUS is peripheral fibrous tissue with inflammatory cell infiltration.

The smallest lesion in our study did not demonstrate ringlike enhancement in the arterial phases (Fig. 3); furthermore, after US-guided biopsy, histopathology determined this lesion to be an SNN, which was missed by CT because the lesion was too small, measuring at just $0.85 \mathrm{~cm}$. It should be noted that the pathological diagnosis of surgically resected specimens is not difficult, but coagulation necrosis, fibrous bands and basically normal hepatocytes must be seen under the microscope in the diagnosis of ultrasound-guided biopsy specimens [25].

In our study, one wedge-shaped lesion demonstrated a blood flow signal in the periphery, and CEUS revealed peripheral and septal enhancement in the arterial phase, which likely became washed out in the portal phase (Fig. 2), and most of the center showed no enhancement. This patient had a history of injury that may be consistent with the SNN hypothesis based on trauma [6]. The following year, the patient underwent surgery at his resident city's hospital; furthermore, postoperative pathology showed the same result as our biopsy and histopathology analysis.

For patients receiving interventional therapy for HCC or hepatic metastasis, CEUS revealed no enhancement with coagulation necrosis resembling SNN, so inquiring about the medical history of each patient is important for diagnosis. For some ovarian SNNs, the mild-moderate thin ring-like enhancement in the arterial phase becomes 
isoenhancement in the portal or late phases with no internal enhancement in any of the three phases, which requires differential diagnosis from metastatic cancer. For some hepatic metastatic lesions, ring-like or peripheral enhancement is observed during the arterial phase but it is inhomogeneous and thicker peripheral enhancement, further changes to hypoenhancement in the portal and late phases. Deniz et al. [26] reported that SNNs in the liver are not always benign, which is important to identify minute foci of metastatic carcinoma. Thus, we should combine the patient's medical history, tumor marker test, and further ultrasound-guided puncture biopsy.

On follow-up with our patients, two SNNs in the liver had regressed, and another two of SNNs had calcified. Choi et al. [27] reported that a hepatic mass (diameter $2 \mathrm{~cm}$ ) was diagnosed as an SNN by liver biopsy, and follow-up for 7 months revealed that the SNN had spontaneously regressed. Thus, conservative treatment and followup should be a good management option for SNNs.

\section{Conclusion}

CEUS is a superior method for detecting SNNs in fatty liver compared to CVUS. Using CEUS, the diagnosis of SNNs in the fatty liver can be made according to lesion shape and enhancement characteristics, and proven by USguided biopsy and histopathology to avoid unnecessary surgery. Overall, CEUS is a valuable tool for the diagnosis of SNNs in fatty liver.

Author contributions Contrast-enhanced ultrasound examination, LPL and XLY; Data Curation, YLP, YJZ, JJL; Formal analysis, YLP, LPL, YJZ, JJL; Investigation and validation, YLP, YJZ, JJL; WritingOriginal Draft preparation, YLP, LPL; Writing-Review and editing, LPL and XLY. Supervision and project administration, XLY; Funding acquisition, LPL.

Funding This study was supported by the National Natural Sciences Foundation of China (Grant No. 81171361).

\section{Declarations}

Conflict of interest Data in our study is availability. All authors have read and agree to the published version of the manuscript. All authors have declared that they have no conflicts of interest regarding this article.

Human and animal rights This article does not contain any studies with animals performed by any of the authors. Each patient in this study consented to participate the research and to using relevant material. This study was approved by the ethics committee of our institutions and had clinical trials registration.
Open Access This article is licensed under a Creative Commons Attribution 4.0 International License, which permits use, sharing, adaptation, distribution and reproduction in any medium or format, as long as you give appropriate credit to the original author(s) and the source, provide a link to the Creative Commons licence, and indicate if changes were made. The images or other third party material in this article are included in the article's Creative Commons licence, unless indicated otherwise in a credit line to the material. If material is not included in the article's Creative Commons licence and your intended use is not permitted by statutory regulation or exceeds the permitted use, you will need to obtain permission directly from the copyright holder. To view a copy of this licence, visit http://creativecommons.org/licenses/by/4.0/.

\section{References}

1. Younossi Z, Tacke F, Arrese M, Sharma BC, Mostafa I, Bugianesi E, Wong VW-S, Yilmaz Y, George J, Fan J, Vos MB (2019) Global perspectives on nonalcoholic fatty liver disease and nonalcoholic steatohepatitis. Hepatology 69(6):2672-2682

2. Boyraz M, Hatipoğlu N, Sarı E, Akçay A, Taşkın N, Ulucan K, Akçay T (2014) Non-alcoholic fatty liver disease in obese children and the relationship between metabolic syndrome criteria. Obes Res Clin Pract 8:e356-363

3. Berardis S, Sokal E (2014) Pediatric non-alcoholic fatty liver disease: an increasing public health issue. Eur J Pediatr 173:131-139

4. Konno K, Ishida H, Sato M, Komatsuda T, Ishida J, Naganuma H, Hamashima Y, Watanabe S et al (2001) Liver tumors in fatty liver: difficulty in ultrasonographic interpretation. Abdom Imaging 26:487-491

5. Liu LP, Dong BW, Yu XL, Liang P, Zhang DK, An LC (2009) Focal hypoechoic tumors of Fatty liver: characterization of conventional and contrast-enhanced ultrasonography. J Ultrasound Med 28:1133-1142

6. Shepherd NA, Lee G (1983) Solitary necrotic nodules of the liver simulating hepatic metastases. J Clin Pathol 36:1181-1183

7. Pananwala H, Pang TC, Eckstein RP, Hudson BJ, Newey A, Samra JS, Hugh TJ (2014) The enigma of solitary necrotic nodule of the liver. ANZ J Surg 84(4):260-265

8. Sundaresan M, Lyons B, Akosa AB (1991) 'Solitary' necrotic nodules of the liver: an aetiology reaffirmed. Gut 32:1378-1380

9. Li DJ, Huang WB, Zhou XJ, Zhang XH, Lu ZF, Ma HH (2005) Solitary necrotic nodules of liver: 2 cases report and review of literature. J Diag Pathol 12(5):342-344

10. Goel G, Rao S, Khurana N, Sarda AK (2014) Solitary necrotic nodule of liver (SNNL): a report of two cases. J Clin Diagn Res 8:115-116

11. Teixeira Martins RJ, Guilherme Tralhão J, Cipriano MA, Castro Sousa F (2014) Solitary necrotic nodule of the liver: a very challenging diagnosis. BMJ Case Rep. https://doi.org/10.1136/ bcr-2013-202364

12. Hwang JY, Lee JE, Jung MJ (2020) A challenging case of solitary necrotic nodules of the liver mimicking hepatic metastases: CT, MRI, and PET-CT findings. J Belg Soc Radiol 104(1):16

13. Chiorean L, Tana C, Braden B, Caraiani C, Sparchez Z, Cui XW, Baum U, Dietrich CF (2016) Advantages and limitations of focal liver lesion assessment with ultrasound contrast agents: comments on the european federation of societies for ultrasound in medicine and biology (EFSUMB) guidelines. Med Princ Pract 25(5):399-407

14. Luo Y, Yue W, Li Z, Wang P (2021) Contrast-enhanced ultrasound and its differential diagnosis in 21 patients with intrahepatic space-occupying lesions under the background of fatty liver. Ann Palliat Med 10(3):3097-3104 
15. Liu LP, Dong BW, Yu XL, Zhang DK, Li X, Li H (2008) Analysis of focal spared areas in fatty liver using color Doppler imaging and contrast-enhanced microvessel display sonography. J Ultrasound Med 27(3):387-394

16. Chen CJ, Chou SC, Chen HJ, Chen HY (2010) Solitary necrotic nodule with larval infestation in the liver on F-18 FDG PET/CT. Clin Nucl Med 35:724-725

17. De Luca M, Luigi B, Formisano C, Formato A, De Werra C, Cappuccio M, Loffredo A, Forestieri P (2000) Solitary necrotic nodule of the liver misinterpreted as malignant lesion: considerations on two cases. J Surg Oncol 74:219-222

18. Yoon KH, Yun KJ, Lee JM, Kim CG (2000) Solitary necrotic nodules of the liver mimicking hepatic metastasis: report of two cases. Korean J Radiol 1:165-168

19. Delis SG, Kelgiorgi DA, Sofianidis AA, Triantopoulou CC, Papailiou IA, Dervenis CG (2009) Solitary necrotic nodule of the liver mimicking hepatocellular carcinoma: a case report. Case J 25(1):85

20. Wang Y, Yu X, Tang J, Li H, Liu L, Gao Y (2007) Solitary necrotic nodule of the liver: contrast-enhanced sonography. J Clin Ultrasound 35:177-181

21. Chunyu Lu, Tang S, Zhang X, Wang Y, Wang K, Shen P (2021) Characteristics of hepatic solitary necrotic nodules on contrastenhanced ultrasonography. BMC Gastroenterol 21(1):35

22. Kim DH, Shim JJ, Kim BH (2013) A solitary necrotic nodule of the liver. Korean J Intern Med 28(4):510-511
23. Francica G, Meloni MF, Riccardi L, de Sio I, Caturelli E, Terracciano F, Giangregorio F, Chiang J, Danzi R, Marra A, Niosi M, Ranalli TV, Pompili M (2021) Contrast-enhanced ultrasound findings in patients with rare solitary necrotic nodule of the liver-a multicenter report. Ultraschall Med. https://doi.org/10. 1055/a-1579-9457

24. Fang J, Ma X, Yu D, Ma X, Xiang Y, Guo L (2017) Specific imaging characteristic of solitary necrotic nodule of the liver: marked peripheral rim-like enhancement with internal hypointensity on longer delayed MRI. Eur Radiol 27(9):3563-3573

25. Ren Gang, Yu Guo, Jin Wei (2007) Clinicopathologic features of solitary necrotic nodules of liver by fine needle aspiration biopsy. Clin J Med Off 35(6):894-896

26. Deniz K, Coban G (2010) Solitary necrotic nodule of the liver: always benign? J Gastrointest Surg 14(3):536-540

27. Choi CS, Cho EY, Jeong JS, Im CJ, Yang BJ, Kim HC (2010) Spontaneous regression of a solitary necrotic nodule of the liver. Hepatol Int 4(3):649-652

Publisher's Note Springer Nature remains neutral with regard to jurisdictional claims in published maps and institutional affiliations. 